\title{
Estudio comparado de los servicios ecosistémicos de los humedales Guarinó y Cauquita, en el Valle del Cauca (Colombia)*
}

Comparative Study of the Ecosystem Services in the Guarinó and Cauquita Wetlands in Valle del Cauca (Colombia)

Saúl Eduardo Henao Echeverria ${ }^{a}$

Universidad Santiago de Cali, Colombia

sehevet@hotmail.com

ORCID: http://orcid.org/0000-0002-4463-3083

Diego Fernando Carabali Altamirano

Universidad Santiago de Cali, Colombia

ORCID: http://orcid.org/0000-0003-3718-6892

Luis Antonio González Escobar

Universidad Santiago de Cali, Colombia

ORCID: http://orcid.org/0000-0003-3000-7708

Luis Carlos Marmolejo

Universidad Santiago de Cali, Colombia

ORCID: http://orcid.org/0000-0003-3051-753X
DOI: https://doi.org/10.11144/Javeriana.ayd22-43.ecse Redalyc: http://www.redalyc.org/articulo.oa? id $=151560179004$

Fecha de recepción: 01 Julio 2018 Fecha de publicación: 30 Diciembre 2018

\section{Resumen:}

Se presenta una comparación de los servicios ecosistémicos de los humedales Guarinó y Cauquita. Para esto, se parte de un diagnóstico ambiental en el que se establecen los principales aspectos físicos, bióticos y económicos, y se caracteriza su problemática general. Los servicios ecosistémicos identificados fueron calificados y comparados en función de su calidad a través de la construcción de un índice de calidad ecológica, soportado en un sistema de indicadores, que permite establecer las relaciones de dependencia entre las distintas acciones que generan impactos negativos en el flujo de servicios ecosistémicos. Se finaliza con una propuesta educativa que busca sensibilizar sobre la problemática e importancia de los humedales. La metodología que se aplica es de enfoque mixto, cualitativo e interpretativo. Los resultados permiten concluir que los humedales en estudio son espacios valiosos para la conservación de biodiversidad y para mantener el acceso a condiciones de bienestar a las poblaciones locales.

Palabras clave: biodiversidad, ecosistemas, humedales, servicios ecosistémicos, bióticos.

\section{Abstract:}

This paper provides a comparison of the ecosystem services between the Guarinó and Cauquita wetlands. To do so, this work starts with an environmental diagnosis that sets the main physical, biotic and economic aspects and characterizes the general problems. The ecosystem services identified were graded and compared according to their quality. To that end, an ecologic quality index was constructed based on an indicator system. It enables to identify some dependence relationships between the different actions having negative impacts on the ecosystem service flow. At the end, this work provides an educative proposal seeking to raise awareness on the problems and importance of the wetlands. The methodology herein is a combined approach, qualitative and interpretative. The results allow concluding that the wetlands under study are valuable spaces to preserve the biodiversity as well as ensuring the local populations their access to well-being.

Keywords: biodiversity, ecosystems, wetlands, ecosystem services, biotic.

Notas de autor:

${ }^{a}$ Autor de correspondencia. Correo electrónico: sehevet@hotmail.com 


\section{Introducción}

La convención de Ramsar (1971) reconoce a los humedales como vitales para la supervivencia humana en razón de los innumerables beneficios o "servicios ecosistémicos" que brindan a las comunidades locales, entre los cuales se encuentran: el suministro de agua dulce, de alimentos y de materiales de construcción; ser soporte de biodiversidad; además de controlar las crecidas de los ríos y recargarse de aguas subterráneas, lo que ayuda a la mitigación del cambio climático.

Sin embargo, las políticas locales permisivas, la expansión urbana, los cambios del uso del suelo para monocultivos y las actividades agrarias de pastoreo han generado cambios en el ecosistema, como desecación, drenaje, deforestación, sedimentación y contaminación.

Este tipo de intervenciones en los ecosistemas de humedal han estado respaldadas por instrumentos de ley por parte de instituciones que deberían velar por la protección del medio ambiente. Una muestra de esto es el Decreto 1180 de 2003, que establece la obtención de licencias ambientales para el cierre de humedales y el cambio de uso del suelo.

La magnitud de dichas afectaciones en el departamento del Valle del Cauca es señalada por Restrepo y Naranjo (1987), quienes indican que para mediados del siglo XX había en el valle geográfico del río Cauca más de 50.000 hectáreas de humedales, los cuales fueron reducidos a menos de 2650 hectáreas para el año 2002, lo que hizo que estos ecosistemas fueran declarados como amenazados por la adecuación de tierras para la expansión agropecuaria y urbana.

Sáenz (2009), Vásquez (2001) Rivera, Naranjo y Duque (2007) y Uribe (2014) corroboran que el monocultivo de caña de azúcar y la adecuación de tierras con fines de urbanización han sido las principales fuerzas de transformación y deterioro de los humedales de la margen izquierda del río Cauca, particularmente, a partir de lo que ellos señalan como la consolidación de una élite política en la ciudad de Cali que logró posicionar un discurso de desarrollo que impulsó el Plan Agua Blanca, el cual permitió la adecuación de tierras que hacían parte de la llanura de inundación del río Cauca, comúnmente designadas como "tierras ejidales", para ser destinadas a la extensión de la agroindustria de la caña de azúcar y a la generación hidroeléctrica, mediante la regulación del caudal del río Cauca.

Existen diversos estudios que señalan las múltiples problemáticas ambientales que enfrentan los ecosistemas de humedal por la intervención del hombre, como: incremento de la frontera urbana, ganadería extensiva, deforestación y sedimentación de los cuerpos de agua, entre otros, y la importancia de conservar estos ambientes (Senhadji-Navarro, 2017).

Todas las acciones antrópicas sobre los humedales tienen efectos negativos, tanto en la biodiversidad silvestre como en las mismas comunidades humanas, ya que se ven afectados los servicios ecosistémicos de los cuales se benefician (Díaz, Fargione, Chapin III y Tilman, 2006, p. 3).

A pesar de esto, la comprensión de estos ecosistemas desde una perspectiva integral aún es incipiente. Quizás el estudio más completo que se ha hecho hasta el presente es la evaluación de los servicios ecosistémicos de la Ciénaga Grande de Santa Marta (Vilardy y González, 2011).

Por esta razón, los objetivos de la presente investigación fueron: 1) realizar un diagnóstico ambiental de los humedales Cauquita y Guarinó, 2) hacer una comparación de la calidad de los servicios ecosistémicos en ambos humedales y 3 ) formular una propuesta educativa para sensibilizar sobre la problemática ambiental de estos ecosistemas y contribuir a su incorporación a nivel curricular. La relevancia de la investigación se basa en la dependencia que tienen las comunidades humanas de la conservación de los ecosistemas y, de manera específica, de los servicios que estos proveen y que se relacionan con su bienestar.

La educación ambiental ha generado los espacios para integrar los fundamentos de las ciencias sociales y naturales que les dan el carácter de interdisciplinar, multidisciplinar y transversal, al relacionar ciencias como la política, la sociología, la psicología y la ecología, con la experiencia y el ejercicio del aula de clase, donde los participantes expresan sus distintos puntos de vista. 
Con esta perspectiva, la presente investigación tiene como objetivo visibilizar la utilidad de estos ecosistemas a partir del análisis de los beneficios que obtienen del uso de la biodiversidad y de los humedales las Comunidades de Robles, Quinamayó y Villapaz, y cómo se ve afectado su bienestar debido al deterioro y la pérdida de los servicios ecosistémicos.

\section{Marco de referencia}

\section{Servicios ecosistémicos: el nexo entre biodiversidad y bienestar humano}

Los servicios ecosistémicos se refieren las contribuciones de la biodiversidad y de los ecosistemas al bienestar humano y al sostenimiento de la vida en el planeta (Ministerio del Medio Ambiente, 2013).

Además, el Sistema de Información Ambiental de Colombia, a través de su página en internet, hace explícita la manera como los seres humanos interactúan con la naturaleza a diferentes escalas de tiempo y espacio, y son ellos quienes constituyen finalmente los servicios en el momento en que establecen usos y significados diferenciados de la naturaleza a través de la cultura (Sistema de Información Ambiental de Colombia, 2019, p. 1).

De esta manera, los servicios ecosistémicos contribuyen al bienestar humano, mediante la posibilidad de acceder a condiciones de seguridad, salud y beneficios materiales, que se traducen en libertad de opción y en autonomía, bases de la cohesión social, el respeto mutuo y la habilidad de ayudar a otros. Estos beneficios o servicios contribuyen directamente a la sociedad, a través de la provisión de alimentos y materiales, o indirectamente, al mantener las condiciones propicias para la conservación de la vida, mediante la formación del suelo, la provisión de agua, la regulación climática e hídrica, la polinización, la recreación o el sentido de pertenencia (Alcaldía de Medellín, 2012).

Se distinguen diferentes categorías y tipologías de servicios, como trabajo y alimentación, entre otras. La clasificación más usada distingue las categorías de: servicios de abastecimiento, de apoyo o soporte, servicios de regulación y servicios culturales. Cada una de estas categorías manifiesta una dimensión específica de la biodiversidad en relación con el bienestar humano, aunque articulada con todas las demás; por lo tanto, el funcionamiento de los ecosistemas depende del mantenimiento de las partes y del todo, lo que determina la capacidad de proveer servicios a la humanidad (Balvanera, 2012; Alcaldía de Medellín, 2012). 


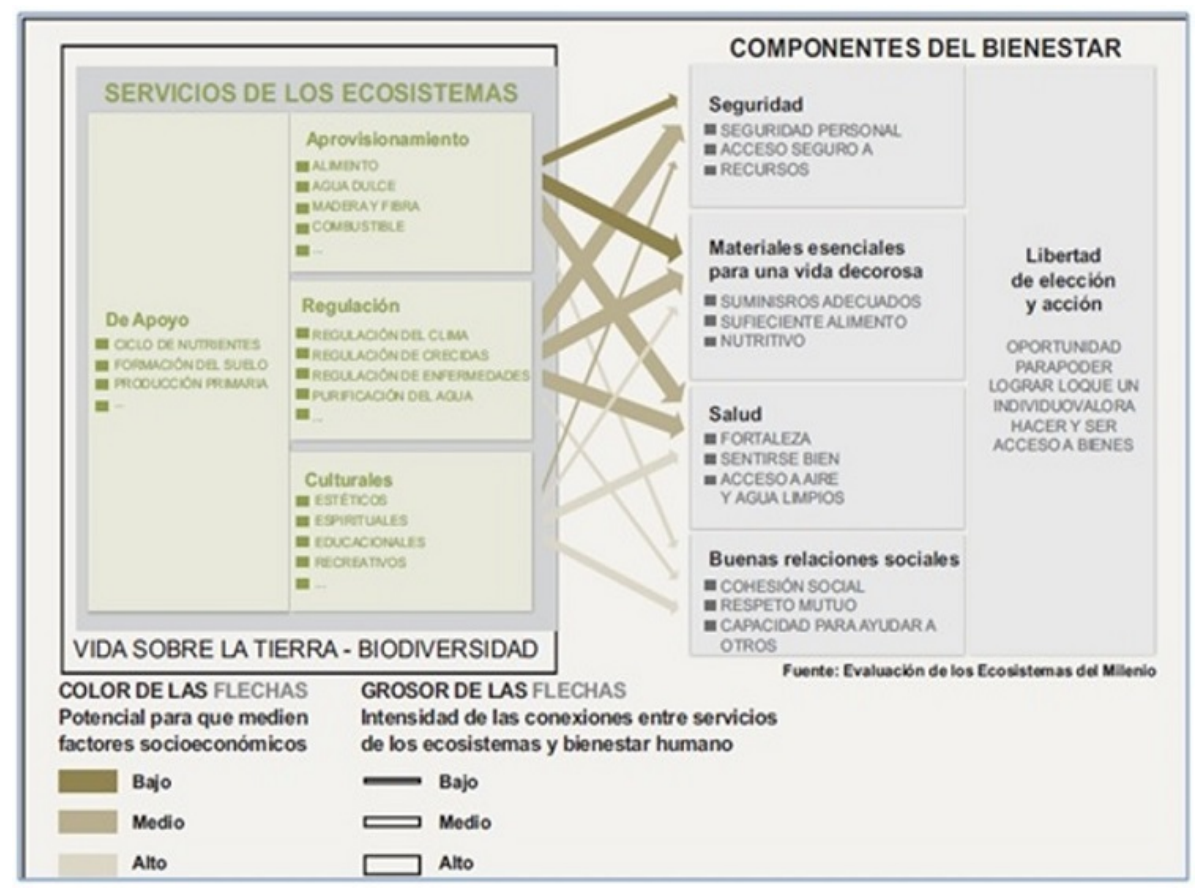

FIGURA 1

Relaciones entre servicios ecosistémicos y bienestar humano

Fuente: Millennium Ecosystem Assessment (2005), traducido por Diego Carabalí y Saúl Henao

En la figura 1 podemos ver la manera como los diferentes servicios ecosistémicos se relacionan con el bienestar humano en diferentes niveles.

\section{Materiales y métodos}

\section{Área de estudio}

Los humedales Cauquita y Guarinó se localizan en jurisdicción de los corregimientos de Robles, Quinamayó y Villapaz, al sur del departamento del Valle del Cauca (Colombia) (figura 2). 


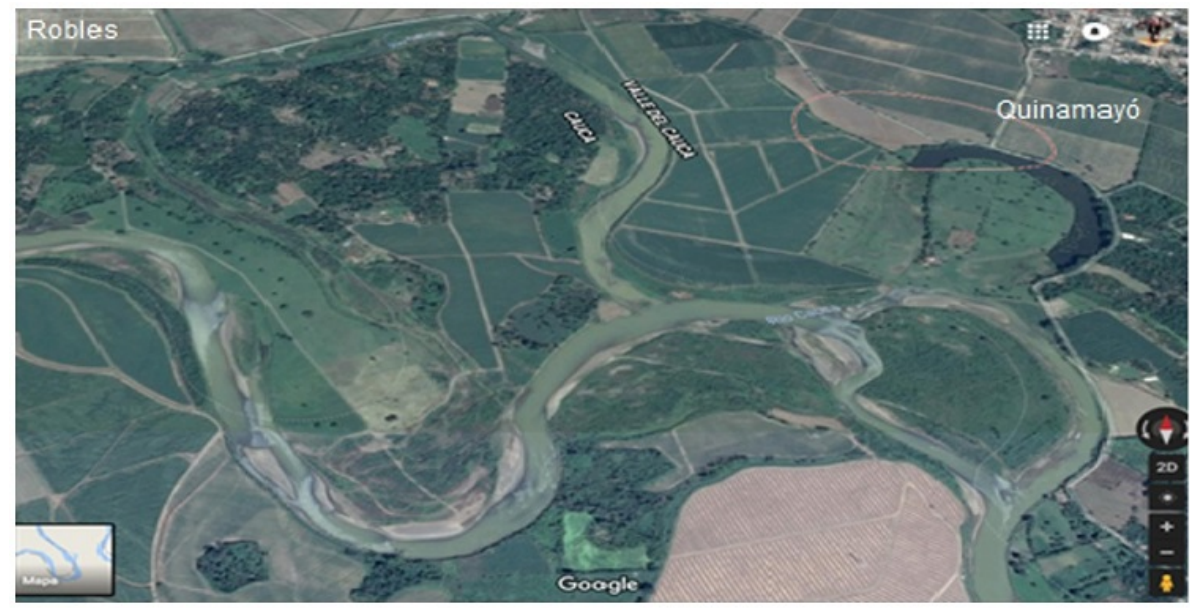

FIGURA 2

Vista aérea de las madreviejas Cauquita y Guarinó

Fuente: Google Maps, http://bit.ly/2KmcuRz

Las principales afectaciones a estos humedales se han dado como consecuencia directa del monocultivo de caña de azúcar, la expansión urbana y la falta de compromiso por parte de los actores involucrados, como la autoridad ambiental, el gremio cañicultor, el gremio de la construcción, la alcaldía municipal y la propia comunidad.

Los impactos negativos de mayor influencia son ocasionados por la desecación de los humedales, el uso intensivo del agua y del suelo, la fragmentación del hábitat y la contaminación con vertimientos de origen doméstico y agrícola que drenan al humedal por escorrentía, lo que causa eutrofización.

Esta situación es más acentuada en el humedal Cauquita, en el cual se encuentra la mayor parte del espejo de agua invadido con vegetación acuática que impide el desarrollo de los procesos fotosintéticos y conlleva un deterioro de los servicios ecosistémicos vinculados con la integridad del paisaje y la belleza escénica.

La población de los corregimientos de Robles, Quinamayó y Villapaz no supera los 15.000 habitantes y corresponde mayoritariamente a comunidades negras descendientes de esclavos africanos que fueron traídos a la zona para trabajar en el establecimiento de haciendas y minas durante el periodo de colonización española (Popó y Ruíz, 2012). Los servicios públicos con mayor cobertura son la energía eléctrica y el acueducto, prestados por la Empresa de Energía del Pacífico-EPSA y Acuasur, respectivamente.

No se cuenta con servicio de alcantarillado y tampoco hay instalaciones de gas natural domiciliario, por lo cual para cocinar se utilizan pipetas con gas propano y en algunos casos fogones de leña. Existen pocas instituciones educativas donde se presta el servicio de básica primaria y bachillerato, la mayoría son públicos; cada corregimiento cuenta con su centro de salud, donde se prestan los servicios básicos asociados a los programas gubernamentales de promoción y prevención (Popó y Ruíz, 2012).

Las fuentes de empleo son escasas en la zona, y la mayor parte de la población vive de la agricultura, el trabajo como jornaleros en fincas e ingenios o como pequeños comerciantes.

Las unidades productivas las constituyen fincas poliproductivas, donde se practica una agricultura de subsistencia y se comercializan los excedentes en plazas de mercado cercanas (10\%) o través de venta directa o a intermediarios (90\%). Aún se conservan prácticas tradicionales, como la diversificación de cultivos, el uso de la hojarasca y de los residuos orgánicos para fertilizar el suelo y la limpieza y preparación del terreno de forma manual utilizando machete y azadón.

En las fincas se encuentran cultivos permanentes, como naranja valencia, tangelo, limón Tahití, limón común, mandarina, guanábana, mango, zapote, arazá, cacao y plátano. También se encuentran cultivos transitorios, como maíz, frijol, yuca, soya, arroz, zapallo, tomate, millo y maracuyá. 
Otra actividad de importancia es la pesca, debido a que es una actividad que mantiene unida a la comunidad y es una fuente de suministro alimenticio a lo largo de la vida del humedal, por lo que es clave para la subsistencia y la seguridad alimentaria.

Actualmente, la mayor parte de la tierra no se encuentra legalizada, sino que su propiedad se establece consuetudinariamente a través del uso y su valor se mide como valor de producción, lo que constituye una de las principales limitaciones en el manejo ambiental de los humedales.

\section{Tipo de investigación}

El diseño de la investigación es de tipo no experimental transversal. Por lo que se recogen los datos en un único momento, sin controlar ningún elemento interno o externo a la investigación (Hernández, Fernández y Baptista, 1991). La perspectiva metodológica se relaciona con la investigación social cualitativa, de carácter mixto, descriptivo e interpretativo, según los lineamientos propuestos por Bonilla-Castro y Rodríguez-Sehk (2005).

Se parte del diseño y registro de categorías temáticas para la recolección, análisis y control de la información. El tipo de análisis de la información es inductivo, es decir que se parte del registro y reconstrucción de datos particulares para llegar a discusiones generales que aporten una perspectiva integral y totalizante.

\section{Métodos y procedimientos}

El diagnóstico de la situación ambiental se realizó con base en información secundaria, mediante la revisión de documentos y estudios técnicos realizados en los humedales, así como en otros ecosistemas en relación con estos. Se usó la técnica de análisis cualitativo de contenido, mediante el diseño de categorías temáticas o de registro, según Varguillas (2006).

Las categorías de registro que se definieron son las siguientes: 1) Espejo lagunar (alteraciones hidrológicas), 2) calidad del agua (presencia de vertimientos), 3) biodiversidad (servicios ecosistémicos), 4) eutrofización (dominancia de vegetación acuática), 5) actividades productivas, 6) infraestructura creada (presencia de canales, diques, motobombas y distritos de riego), 7) actividades recreativas, 8) estatus de conservación (existencia de planes de manejo) y 9) población (demografía).

Se realizaron cinco visitas de campo, en las cuales se obtuvo información mediante registro fotográfico y la aplicación de entrevistas y cuestionarios semiestructurados a informantes claves. Las entrevistas y cuestionarios se efectuaron una vez que se obtuvo el consentimiento de los participantes.

La comparación de la calidad de los servicios ecosistémicos de los humedales Cauquita y Guarinó se hizo primero mediante la identificación de los estos servicios, la cual se realizó por medio de listas de chequeo, aplicadas y verificadas en campo a través de observación y registro fotográfico. Las listas de chequeo utilizadas se basaban en la clasificación de la Common International Classification of Ecosystem Services (CICES) (Haines-Young y Potschin, 2017), y fueron cotejadas y complementadas con los listados de Millennium Ecosystem Assessment (2005), De Groot, Wilson y Boumans (2002) y Vilardy y González (2011). La aplicación de listas de chequeo utiliza una serie de planillas con información soportada en bases documentadas, lo que hace de ellas un instrumento confiable (Arana, 2015).

Una vez identificados y caracterizados los servicios ecosistémicos generados en ambos humedales, se procedió a su priorización, para posteriormente comparar su calidad. La priorización se realizó aplicando una ecuación de ponderación, en la que se tuvieron en cuenta las variables de estado, la tendencia de cambio del servicio y su grado de conocimiento (tabla 1). 
TABLA 1

Medición y asignación de pesos para priorizar los servicios ecosistémicos

\begin{tabular}{llllll}
\hline \multicolumn{2}{c}{ Estado del servicio $(\mathrm{E})$} & \multicolumn{2}{c}{ Tendencia del servicio $(\mathrm{T})$} & \multicolumn{2}{c}{ Grado de conocimiento $(\mathrm{C})$} \\
\hline Medición & Asignación & Medición & Asignación & Medición & Asignación \\
\hline Alto & $\mathrm{A}=5$ & $\begin{array}{l}\text { Empeora del } \\
\text { servicio }\end{array}$ & $\mathrm{ES}=5$ & Aceptable & $\mathrm{A}=5$ \\
\hline Medio & $\mathrm{M}=4$ & $\begin{array}{l}\text { Tendencia a } \\
\text { empeorar }\end{array}$ & $\mathrm{TE}=4$ & Escaso & $\mathrm{E}=3$ \\
\hline Bajo & $\mathrm{B}=3$ & Sin tendencia & $\mathrm{S}=3$ & Nulo & $\mathrm{N}=1$ \\
\hline Desconocido & $\mathrm{I}=2$ & $\begin{array}{l}\text { Tendencia a } \\
\text { mejorar }\end{array}$ & $\mathrm{TM}=2$ & & \\
\hline Inexistente & $\mathrm{D}=1$ & $\begin{array}{l}\text { Mejora del } \\
\text { servicio. }\end{array}$ & $\mathrm{MS}=1$ & & \\
\hline
\end{tabular}

Fuente: adaptado de IGCP 604 (s. f.)

La priorización se hace con aquellos servicios cuyas características tienden a deteriorarse y que tienen una alta demanda. Tendrán mayor peso aquellos servicios cuyo estado tienda a empeorar. Luego, tendrán prioridad aquellos que son producidos en gran medida por el humedal. Por último, se le dará mayor prioridad a aquellos servicios de los que se tenga un grado de conocimiento aceptable.

El resultado de la ecuación de priorización (ecuación 1) varía entre 1 y 5 . Si se acerca más a 5 , se hace prioritaria su atención (tabla 2).

Ecuación de priorización:

$$
P=0,35 * E+0,45 * T+0,2 * G
$$

TABLA 2

Asignación de valores para la priorización de servicios

\begin{tabular}{lrl}
\hline Color & Valor & Priorización \\
\hline & $>4 \leq 5$ & Alta \\
\hline & $>3 \leq 4$ & Media \\
\hline & $1 \leq 3$ & Baja \\
\hline
\end{tabular}


La calificación de la calidad de los servicios se realizó mediante el cálculo de un índice de calidad soportado en un sistema de indicadores presión-estado-respuesta. La aplicación de esta metodología está diseñada para ser valorada y validada de manera expedita y participativa a partir de la información suministrada por informantes clave, en este caso investigadores y líderes ambientales comunales con conocimiento suficiente de los humedales.

El proceso se realizó por medio de la ejecución de talleres participativos en campo, en los cuales, una vez explicado el instrumento y el objeto de la evaluación, se pidió la colaboración de los participantes para suministrar información sobre factores y tendencias que inducen cambios y describen el estado del humedal, tales como caracterización física, biótica, económica, administrativa y sociocultural, lo cual fue complementado con registro fotográfico, recorridos guiados y revisión bibliográfica.

La aplicación del instrumento se basó en la investigación mixta de carácter cualitativo e interpretativo a través del uso de herramientas didácticas y participativas del diagnóstico rural participativo (DRP) que favorecen un diálogo e intercambio de saberes entre los investigadores y la comunidad (Expósito-Verdejo, 2003).

La construcción del indice de calidad fue una aproximación numérica de la interacción entre servicios ecosistémicos y factores que afectan el estado de estos y de los humedales en general. La información de la cual se dispuso fueron registros parciales o puntuales, no permanentes ni dinámicos, los cuales prácticamente fueron estimados.

Por lo tanto, la metodología aplicada fue analítica y permitió determinar las relaciones de dependencia entre las distintas categorías involucradas, de las cuales se obtuvieron indicadores para hacer una calificación ecológica en función directa de su probabilidad de darse, su duración, desarrollo y magnitud, que son los criterios mínimos empleados para la caracterización de los factores que pueden afectar el estado de los humedales.

Debido a los riesgos asociados con la presencia o probabilidad, todos los factores están afectando los humedales, por lo tanto, se afecta la calificación ecológica (tabla 3), y como se dan diferencias en la importancia relativa entre cada una de ellas, se aplica un factor de ponderación que explica esas diferencias, obtenido de manera subjetiva por el investigador y los profesionales especializados con la colaboración de líderes comunitarios. 
TABLA 3

Calificación ecológica

\begin{tabular}{lr}
\hline $\begin{array}{c}\text { Calificación (Ce) } \\
\text { Ecológica }\end{array}$ & \multicolumn{1}{c}{ Calificación } \\
\hline Muy alta & $8,0-10,0$ \\
\hline Alta & $6,0-7,9$ \\
\hline Media & $4,0-5,9$ \\
\hline Baja & $2,0-3,9$ \\
\hline Muy baja & $0,0-1,9$ \\
\hline
\end{tabular}

Fuente: Arana (2015)

Para calificar la calidad de los servicios que proveen los humedales, se realiza la sumatoria de los resultados de todos los indicadores y a ese valor se le saca el promedio, para lo cual se divide entre el total de indicadores, que en nuestro caso son 11 (ecuación 2).

$$
\text { Ce total }=(\Sigma \mathrm{Ce}) / \text { total de indicadores }
$$

Una vez establecidas las acciones impactantes en el humedal y los servicios impactados, se procede a la identificación de los principales impactos ambientales tipo causa-efecto. Como se trata de un método multidisciplinario, se identifican los factores críticos, que requieren un análisis más profundo, con respecto a aquellas acciones productivas que generan impactos ambientales de importancia.

El índice de calificación ecológica (Ce) califica las consecuencias del impacto ambiental sobre la calidad físico-biótica y socioeconómica del medio ambiente, expresado por la suma ponderada de cinco factores o criterios característicos: clase, presencia o probabilidad, desarrollo, duración y magnitud relativa del impacto (tabla 4). 
TABLA 4

Valoración de los impactos que inducen cambios en los servicios ecosistémicos

\begin{tabular}{|c|c|c|c|}
\hline Parámetro & Descripción & Rango & Calificación \\
\hline $\begin{array}{l}\text { Presencia o } \\
\text { probabilidad (Pr) }\end{array}$ & $\begin{array}{l}\text { Califica la certeza o probabilidad de que el } \\
\text { impacto pueda ocurrir o presentarse. }\end{array}$ & Cierto & 1,0 \\
\hline \multirow{5}{*}{ Desarrollo (De) } & Califica el tiempo que el impacto tarda en & Muy rápido: $\leq 1$ mes & $0,9-1,0$ \\
\hline & desarrollarse completamente, o sea, la forma en & Rápido: $>1 \leq 6$ meses & $0,7-0,8$ \\
\hline & que evoluciona el impacto, desde que se inicia y & Medio: $>6 \leq 12$ meses & $0,5-0,6$ \\
\hline & manifiesta hasta que se hace presente & Lento: $>12 \leq 24$ meses & $0,3-0,4$ \\
\hline & plenamente con todas sus consecuencias. & Muy lento: $>24$ meses & $0,1-0,2$ \\
\hline \multirow{9}{*}{ Magnitud (Ma) } & Representa la incidencia de la acción causal & Muy alta: $81 \%-100 \%$ & $9-10$ \\
\hline & sobre el factor impactado en el área en la que se & Alta: $61 \%-80 \%$ & $7-8$ \\
\hline & produce el efecto. & Media: $41 \%-60 \%$ & $5-6$ \\
\hline & La magnitud del impacto depende de su & Baja: $21 \%-40 \%$ & $3-4$ \\
\hline & Desarrollo. & Muy baja: $0 \%-20 \%$ & $1-2$ \\
\hline & Califica la dimensión o tamańo del cambio & & \\
\hline & ambiental directo o indirecto (relativo) & & \\
\hline & producido sobre un determinado recurso o & & \\
\hline & $\begin{array}{l}\text { elemento del ambiente, o sobre un bien o un } \\
\text { servicio de la comunidad. }\end{array}$ & & \\
\hline \multirow{5}{*}{ Duración (Du) } & Califica el periodo de existencia. Se refiere a la & Muy larga: $>10$ ańos & $>10$ \\
\hline & valoración temporal que permite estimar el & Larga: $>7 \leq 10$ ańos & $7-9$ \\
\hline & período durante el cual las repercusiones serán & Media: $>4 \leq 7$ ańos & $4-6$ \\
\hline & detectadas en el factor afectado. & Corta $>1 \leq 4$ años & 3 \\
\hline & & Muy corta $\leq 1$ año & $<3$ \\
\hline \multirow[b]{2}{*}{ Clase } & Define el sentido del impacto ambiental & Benéfico & + \\
\hline & $\begin{array}{l}\text { producido por una actividad o acción del } \\
\text { proyecto. }\end{array}$ & Perjudicial & $\cdot$ \\
\hline \multirow{7}{*}{$\begin{array}{l}\text { Calificación } \\
\text { ecológica }(\mathrm{Ce})\end{array}$} & Es la expresión numérica de la interacción de los & Muy alta: $>9$ & $9-10$ \\
\hline & parámetros o criterios. El valor de Ce se & Alta: $>7 \leq 9$ & $7-8$ \\
\hline & corresponde con un valor global de la & Media: $>5 \leq 7$ & $5-6$ \\
\hline & importancia del impacto. Se aplica según la & Baja: $>3 \leq 5$ & $3-4$ \\
\hline & fórmula expuesta (fórmula 1). Califica las & Muy baja: $\leq 3$ & $1-2$ \\
\hline & consecuencias del impacto ambiental sobre la & & \\
\hline & $\begin{array}{l}\text { calidad físico-biótica y socioeconómica del } \\
\text { medio ambiente. }\end{array}$ & & \\
\hline
\end{tabular}

Fuente: adaptado de González, Ramírez, Benítez y Moreno (2014)

\section{Formula de calificación ecológica (Ce)}

La fórmula de calificación ecológica que se aplicó fue la utilizada por la Asociación Colombiana de Ingeniería Sanitaria y Ambiental (Acodal, 1991), la cual se expresa como sigue (ecuación 3):

$$
C e=P r *(\mathrm{a} * D e * M a)+(\mathrm{b} * D u)
$$

Según ella, la calificación ecológica es función de la presencia, el desarrollo, la magnitud y la duración del impacto que se genera por la actividad productiva; donde a y b son factores de ponderación con un valor entre cero y uno, los cuales fueron obtenidos subjetivamente por los especialistas consultados, de modo que se les 
asignó un valor de 0,7 y 0,3 , respectivamente. Con base en esta fórmula se establecieron los valores y se realizó la calificación de la calidad de los servicios que proveen los humedales.

Finalmente, se formuló una propuesta educativa enfocada en los colegios de la zona, específicamente en la población estudiantil del nivel básico y medio. Se escogió esta población como enfoque de la propuesta debido a que existe un alto grado de desconocimiento de los humedales, sus beneficios y su problemática. Asimismo, se consideró a este grupo poblacional por su potencial trasformador a través de plataformas específicas existentes, como los proyectos ambientales escolares (PRAES). El objetivo de la propuesta era sensibilizar a los estudiantes sobre la problemática de los humedales e incorporarla a nivel curricular.

La propuesta educativa considera estrategias específicas, como salidas pedagógicas, conformación de semilleros de investigación y creación de medios y mecanismos de comunicación, difusión y participación que ayuden a vincular de una manera más directa a los estudiantes con los humedales, sus problemáticas y las contribuciones de estos ecosistemas al bienestar humano a través de la provisión de servicios ecosistémicos.

\section{Resultados}

La aplicación del instrumento permitió priorizar once servicios ecosistémicos (tablas 5 y 6 ) de acuerdo a su tendencia de cambio, su estado y su grado de conocimiento. De estos servicios, seis pertenecen a la categoría de regulación y soporte, dos a la categoría de abastecimiento y tres a la categoría de servicios culturales.

Si bien el humedal Guarinó presenta mejores condiciones ecológicas, lo que se refleja en que únicamente cuatro de los servicios tienen una prioridad alta, también se observa que gran parte de los servicios tienen una prioridad media, y, al compartir factores de tensión con Cauquita, existe riesgo de que sus servicios tiendan a empeorar, por lo cual la calificación de la calidad ecológica de los humedales tomó como referencia los once servicios priorizados en Cauquita.

La tabla 5 analiza las variables concernientes a la comparación y evaluación de los servicios ecosistémicos en el humedal y los clasifica a partir de indicadores, se detalla además el impacto ambiental y social en torno al humedal. 
TABLA 5

Servicios ecosistémicos de los humedales Cauquita y Guarinó

\begin{tabular}{|c|c|c|c|}
\hline \multirow{2}{*}{ Categoría } & \multirow{2}{*}{ Bienes y servicios } & \multirow{2}{*}{$\begin{array}{l}\text { Chequeo } \\
\text { Cauquita } \\
\end{array}$} & \multirow{2}{*}{$\begin{array}{l}\text { Chequeo } \\
\text { Guarinó }\end{array}$} \\
\hline & & & \\
\hline \multirow{6}{*}{ 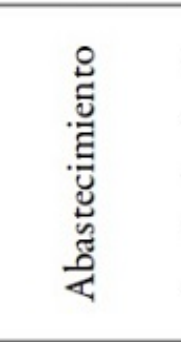 } & Provisión de alimento & Sí & Sí \\
\hline & Madera y fibra & Sí & Sí \\
\hline & Combustible (leña) & Sí & Sí \\
\hline & Recursos genéticos & Sí & Sí \\
\hline & Medicinas naturales & Sí & Sí \\
\hline & Suministro de agua & Sí & Sí \\
\hline \multirow{5}{*}{ 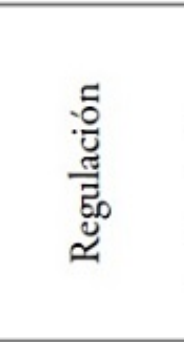 } & $\begin{array}{l}\text { Sujeción de suelo (prevención y control de } \\
\text { procesos erosivos) }\end{array}$ & Sí & Sí \\
\hline & Regulación hídrica & Sí & Sí \\
\hline & Control de plagas & Sí & Sí \\
\hline & Calidad de agua & Sí & Sí \\
\hline & Regulación de nutrientes & Sí & Sí \\
\hline \multirow{2}{*}{ 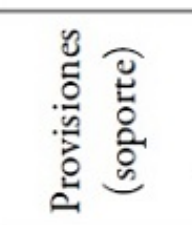 } & $\begin{array}{l}\text { Función de refugio (provisión de hábitat, } \\
\text { mantenimiento de interacciones } \\
\text { biológicas) }\end{array}$ & Sí & Sí \\
\hline & Mantenimiento de la biodiversidad & Sí & Sí \\
\hline \multirow{6}{*}{$\frac{\text { ્ㅗㄹ }}{\stackrel{\Xi}{\Xi}}$} & Inspirativo & No & Sí \\
\hline & Herencia cultural & No & Sí \\
\hline & Ecoturismo & No & Sí \\
\hline & Educación y sensibilización ambiental & Sí & Sí \\
\hline & Disfrute estético (belleza escénica) & No & Sí \\
\hline & Investigación & Sí & Sí \\
\hline
\end{tabular}


TABLA 6

Priorización de los servicios de los humedales Cauquita y Guarinó

\begin{tabular}{|c|c|c|c|c|c|c|c|c|c|c|c|}
\hline \multicolumn{12}{|c|}{ Ecuación de ponderación $\mathrm{P}=0,35^{*} \mathrm{E}+0,45^{*} \mathrm{~T}+0,2^{*} \mathrm{GC}$} \\
\hline \multirow{2}{*}{$\begin{array}{l}\text { Tipo de } \\
\text { Servicio }\end{array}$} & \multirow{2}{*}{ Bienes y servicios } & \multicolumn{4}{|c|}{ Valor Cauquita } & \multirow{2}{*}{ Prioridad } & \multicolumn{4}{|c|}{ Valor Guarinó } & \multirow{2}{*}{ Prioridad } \\
\hline & & $\mathrm{E}$ & $\mathrm{T}$ & GC & $P$ & & $\mathrm{E}$ & $\mathrm{T}$ & GC & $P$ & \\
\hline \multirow{6}{*}{ 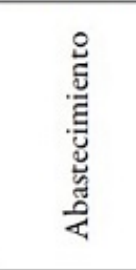 } & Provisión de alimento & 5 & 4 & 5 & 4,55 & Alta & 5 & 4 & 5 & 4,55 & Alta \\
\hline & Madera y fibra & 4 & 3 & 5 & 3,75 & Media & 4 & 3 & 5 & 3,75 & Media \\
\hline & Combustible (leña) & 3 & 3 & 5 & 3,40 & Media & 3 & 3 & 5 & 3,40 & Media \\
\hline & Recursos genéticos & 4 & 4 & 3 & 3,80 & Media & 4 & 4 & 3 & 3,80 & Media \\
\hline & Medicinas naturales & 4 & 3 & 3 & 3,35 & Media & 4 & 3 & 3 & 3,35 & Media \\
\hline & Suministro de agua & 3 & 5 & 5 & 4,30 & Alta & 4 & 4 & 5 & 4,20 & Alta \\
\hline \multirow{4}{*}{ 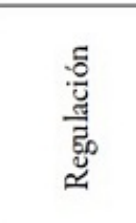 } & Regulación hídrica & 3 & 5 & 5 & 4,30 & Alta & 4 & 4 & 5 & 4,20 & Alta \\
\hline & Control de plagas & 3 & 5 & 3 & 3,90 & Media & 3 & 4 & 3 & 3,45 & Media \\
\hline & Calidad de agua & 3 & 5 & 5 & 4,30 & Alta & 4 & 3 & 5 & 3,75 & Media \\
\hline & $\begin{array}{l}\text { Regulación de nutrientes } \\
\text { en el agua }\end{array}$ & 3 & 5 & 5 & 4,30 & Alta & 3 & 3 & 5 & 3,40 & Media \\
\hline \multirow{3}{*}{ 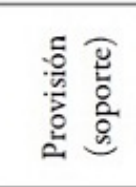 } & Fertilidad del suelo & 5 & 4 & 3 & 4,15 & Alta & 5 & 4 & 3 & 4,15 & Alta \\
\hline & Refugio y hábitat & 3 & 5 & 5 & 4,30 & Alta & 4 & 3 & 5 & 3,75 & Media \\
\hline & $\begin{array}{l}\text { Mantenimiento de la } \\
\text { biodiversidad }\end{array}$ & 3 & 5 & 5 & 4,30 & Alta & 4 & 3 & 5 & 3,75 & Media \\
\hline \multirow{6}{*}{ 氶 } & Inspirativo & 3 & 5 & 3 & 3,90 & Media & 4 & 2 & 3 & 2,90 & Baja \\
\hline & Herencia cultural & 3 & 5 & 5 & 4,30 & Alta & 4 & 4 & 3 & 3,80 & Media \\
\hline & Ecoturismo & 3 & 3 & 5 & 3,40 & Media & 3 & 3 & 3 & 3,00 & Media \\
\hline & Ambiental & 3 & 5 & 5 & 4,30 & Alta & 3 & 3 & 3 & 3,00 & Media \\
\hline & $\begin{array}{l}\text { Disfrute estético (belleza } \\
\text { escénica) }\end{array}$ & 3 & 5 & 5 & 4,30 & Alta & 4 & 3 & 3 & 3,35 & Media \\
\hline & Investigación & 3 & 3 & 3 & 3,00 & Media & 3 & 2 & 3 & 2,55 & Baja \\
\hline
\end{tabular}

Fuente: Arana (2015)

\section{Evaluación y comparación de los servicios ecosistémicos}

En la tabla 7 se analizan las variables concernientes a la comparación y evaluación de los servicios ecosistémicos en el humedal y se clasifican a partir de indicadores; también se detalla el impacto ambiental y social en torno al humedal.

La calificación ecológica de los humedales Cauquita y Guarinó refleja las diferencias en el estado de conservación e integridad del paisaje, que son apreciables mediante la observación y el registro fotográfico. 
TABLA 7

Calificación ecológica de los impactos que afectan el suministro de servicios ecosistémicos en los humedales Cauquita y Guarinó

\begin{tabular}{|c|c|c|c|c|c|c|c|c|c|c|c|c|c|c|c|c|c|c|c|c|}
\hline \multirow{3}{*}{$\begin{array}{l}\text { Categoria de } \\
\text { servicio }\end{array}$} & \multirow{3}{*}{$\begin{array}{l}\text { Servicios } \\
\text { coosistémicos }\end{array}$} & \multirow{3}{*}{ Impactos } & \multicolumn{18}{|c|}{ Calificación ccológica $(\mathrm{Ce})-\mathrm{Ce}=\operatorname{Pr}\left(\mathrm{a}^{*} \mathrm{Dc} c^{*} \mathrm{Ma}\right)+\left(\mathrm{b}^{*} \mathrm{Du}\right)$} \\
\hline & & & \multicolumn{9}{|c|}{ Cauquita } & \multicolumn{9}{|c|}{ Guarinó } \\
\hline & & & $\mathrm{Cl}$ & $\operatorname{Pr}$ & De & 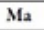 & $\mathrm{Du}$ & a & b & Total & Calificación & $\mathrm{Cl}$ & $\operatorname{Pr}$ & De & $\mathrm{Ma}$ & Du & a & b & Total & Calificación \\
\hline \multirow{2}{*}{ Abastecimiento } & Provisión de alimento & $\begin{array}{l}\text { Pérdida de soberania y } \\
\text { seguridad alimenticia }\end{array}$ & - & 1 & 1 & 7 & 8 & 0,7 & 0,3 & 7,3 & Media & . & 1 & 1 & 7 & 8 & 0,7 & 0,3 & 7,3 & Alta \\
\hline & Suministro de agua & $\begin{array}{l}\text { Agotamiento del recurso } \\
\text { hidrico }\end{array}$ & - & 1 & 1 & 8 & 7 & 0,7 & 0.3 & 7,7 & Alta & $\cdot$ & 1 & 1 & 5 & 8 & 0,7 & 0.3 & 5.9 & Media \\
\hline \multirow{4}{*}{ Regulación } & Fertilidad del suelo & Uso intensivo del suclo & - & 1 & 1 & 7 & 9 & 0,7 & 0,3 & 7,6 & Alta & - & 1 & 1 & 7 & 9 & 0.7 & 0,3 & 7,6 & Alta \\
\hline & Regulación hídrica & $\begin{array}{l}\text { Alteración del flujo } \\
\text { hidrico }\end{array}$ & - & 1 & 1 & 10 & 7 & 0,7 & 0,3 & 9,1 & Muy alta & - & 1 & 0 & 6 & 7 & 0,7 & 0,3 & 6,3 & Alta \\
\hline & Calidad de agua & $\begin{array}{l}\text { Vertimientos de origen } \\
\text { doméstico }\end{array}$ & - & 1 & 1 & 9 & 6 & 0.7 & 0.3 & 8,1 & Muy alta & - & 1 & 1 & 5 & 7 & 0.7 & 0.3 & 5.6 & Media \\
\hline & $\begin{array}{l}\text { Regulación de } \\
\text { nutrientes en el agua }\end{array}$ & $\begin{array}{l}\text { Eutrofización } \\
\text { (proliferación de } \\
\text { veggetación acuatica) }\end{array}$ & - & 1 & 1 & 9 & 7 & 0,7 & 0,3 & 8,4 & Muy alta & - & 1 & 1 & 4 & 7 & 0,7 & 0,3 & 4,9 & Baja \\
\hline \multirow{2}{*}{ Soporte } & Refugio y habitad & $\begin{array}{l}\text { Fraggmentación y } \\
\text { deterioro del hábitat }\end{array}$ & - & 1 & 1 & 9 & 9 & 0,7 & 0,3 & 9 & Muy alta & - & 1 & 0 & 6 & 9 & 0,7 & 0,3 & 6,9 & Alta \\
\hline & $\begin{array}{l}\text { Mantenimiento } \\
\text { biodiversidad }\end{array}$ & Pérdida de especies útiles & - & 1 & 1 & 7 & 8 & 0,7 & 0,3 & 7,3 & Alta & - & 1 & 0 & 5 & 8 & 0,7 & 0,3 & 5,9 & Media \\
\hline \multirow{3}{*}{ Cultural } & Herenciz cultural & $\begin{array}{l}\text { Desarraigo y pérdida de } \\
\text { la identidad territorial }\end{array}$ & - & 1 & 1 & 7 & 8 & 0,7 & 0,3 & 7,3 & Alta & $\cdot$ & 1 & 0 & 5 & 8 & 0,7 & 0,3 & 5,9 & Media \\
\hline & $\begin{array}{l}\text { Educación y } \\
\text { sensibilización }\end{array}$ & $\begin{array}{l}\text { Carencia de conciencia y } \\
\text { compromiso ambiental }\end{array}$ & - & 1 & 1 & 8 & 9 & 0,7 & 0,3 & 8,3 & Muy alta & $\cdot$ & 1 & 0 & 7 & 9 & 0,7 & 0,3 & 7,6 & Alta \\
\hline & $\begin{array}{l}\text { Disfrute ostético } \\
\text { (belleza escénica }\end{array}$ & $\begin{array}{l}\text { Desvalorización de La } \\
\text { naturaleza }\end{array}$ & - & 1 & 1 & 8 & 8 & & & 8 & Alta & - & 1 & 0,2 & 6 & 8 & & & 6,6 & Media \\
\hline $\begin{array}{l}\text { Total } \\
\text { calificación }\end{array}$ & & & & & & & & & & 8,6 & Muy alta & & & & & & & & 6,4 & Media \\
\hline
\end{tabular}

Fuente: elaboración propia, 2018

Se obtuvo una calificación total alta de los impactos que afectan el humedal Cauquita, lo que e corresponde a una calidad ecológica menor. En comparación, el humedal Guarinó presenta una calidad ecológica mayor, que se refleja en una calificación baja de los impactos.

El deterioro de la calidad ecológica en el humedal Cauquita es causado por la presencia, magnitud y permanencia de impactos como la alteración del flujo hídrico, el uso intensivo del suelo, el agotamiento del recurso hídrico, el vertimiento de efluentes agrícolas y domésticos, la fragmentación y el deterioro del hábitat, la eutrofización y la pérdida de especies útiles (Rojas, 2015, p. 5).

Los impactos que más contribuyen al deterioro de la calidad ecológica y del suministro de servicios ecosistémicos son la alteración del flujo hídrico, el vertimiento de aguas residuales, la eutrofización, la fragmentación y el deterioro del hábitat.

De igual manera, se puede observar que el deterioro de las funciones ecosistémicas que inducen cambios a nivel del paisaje afectan la percepción social de las personas, lo que se puede constatar con el deterioro de servicios de tipo cultural, en los que también se aprecian diferencias entre los dos humedales.

En las figuras 3, 4, 5 y 6 se pueden observar los cambios que se han generado en el transcurso de cinco años en el humedal Cauquita y en el humedal Guarinó. 


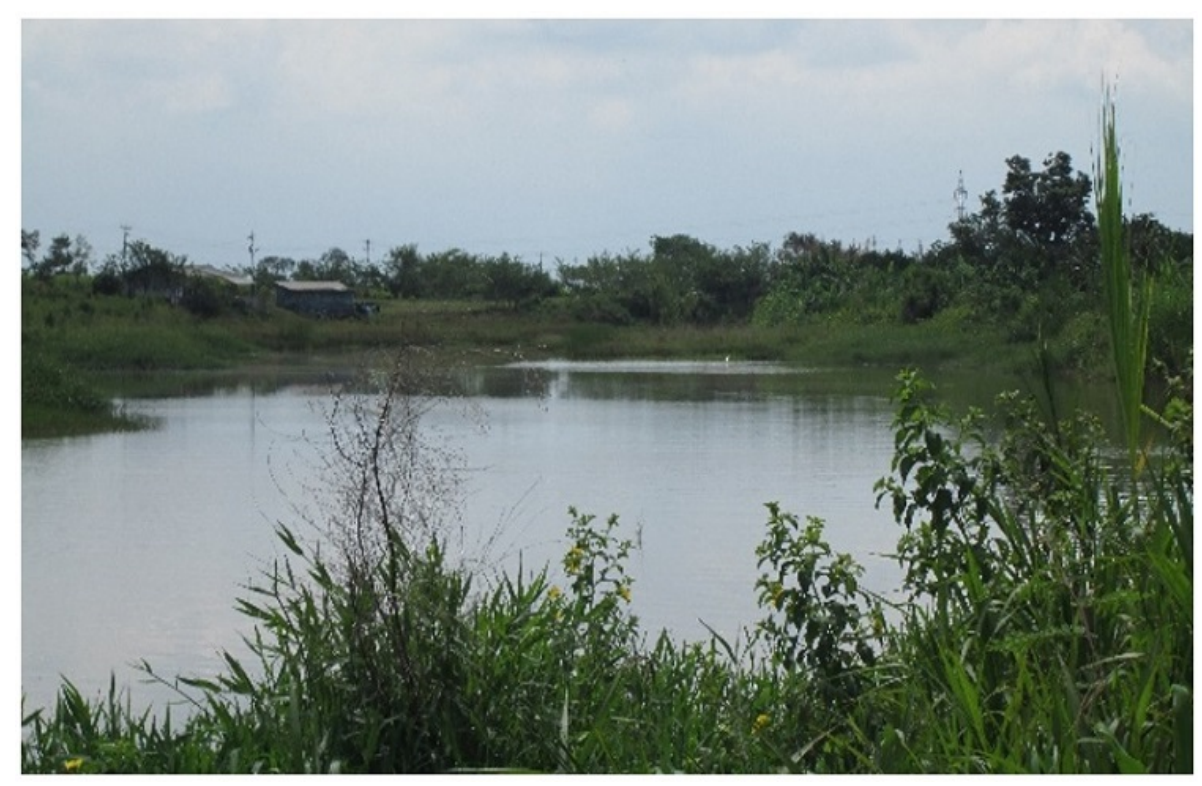

FIGURA 3

Humedal Cauquita

Fuente: fotografía tomada por Saúl Henao, 2013

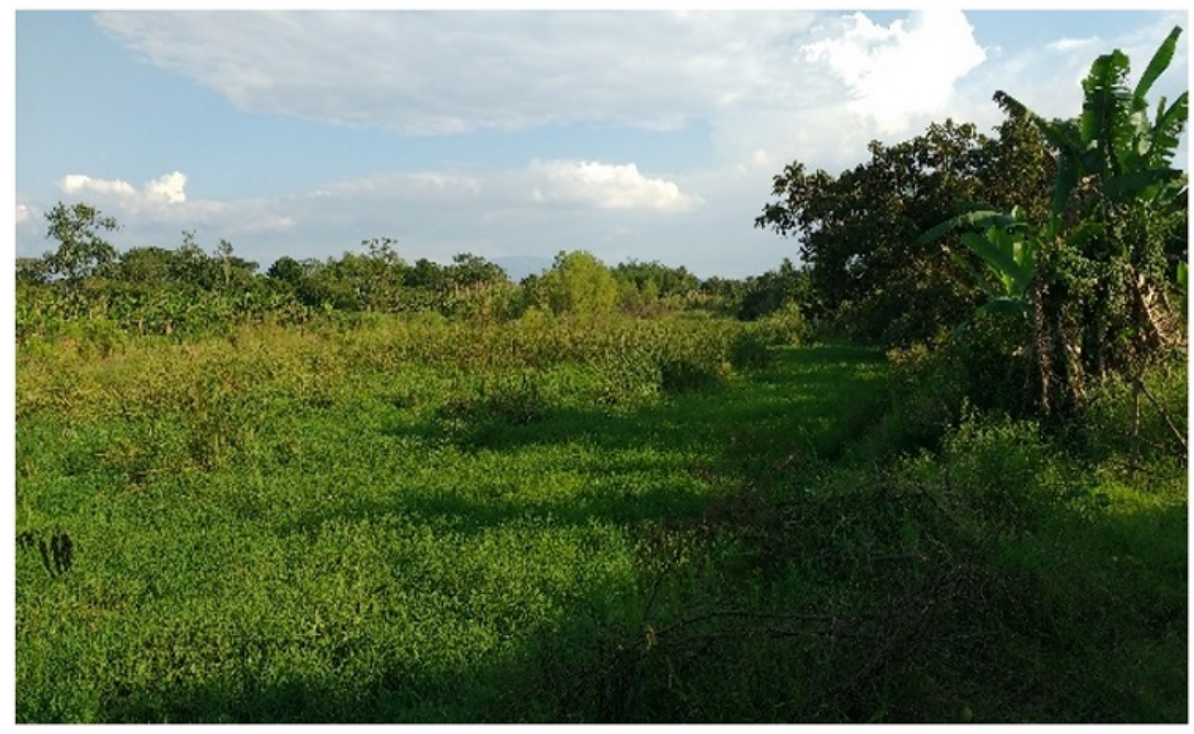

FIGURA 4

Humedal Cauquita

Fuente: fotografía tomada por Saúl Henao, 2018 


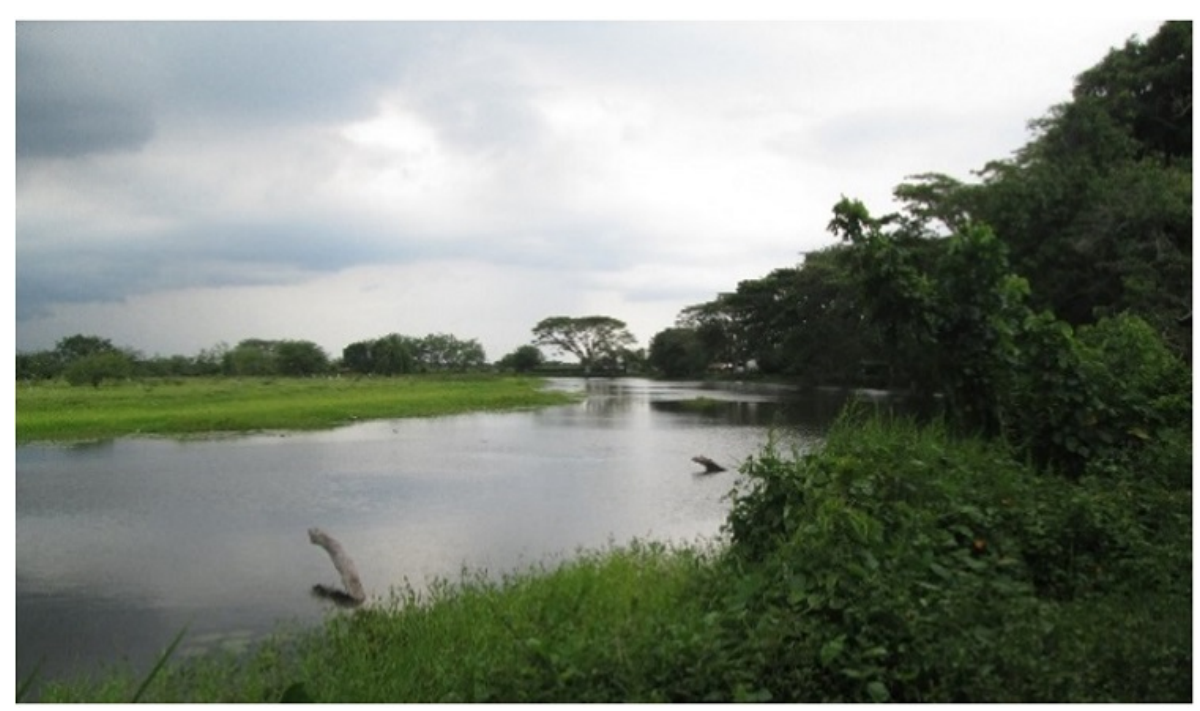

FIGURA 5

Humedal Guarinó

Fuente: fotografía tomada por Saúl Henao, 2013

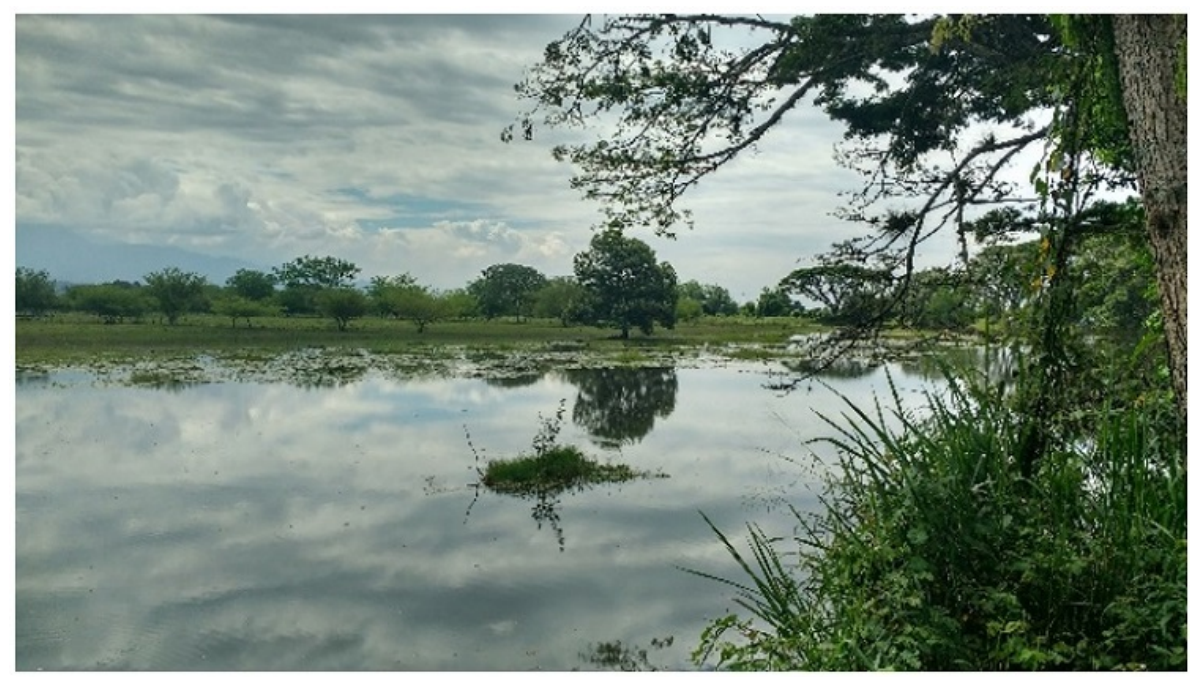

FIGURA 6

Humedad Guarinó

Fuente: fotografía tomada por Saúl Henao, 2018

\section{Propuesta de estrategias educativas e investigativas para la educación formal en la comunidad}

En la tabla 8 se propone una estrategia educativa como parte de los objetivos de este trabajo. En ella se pueden precisar tres estrategias claras, cada una con sus objetivos específicos. Se definen las estrategias y los indicadores utilizados para el cumplimiento de cada uno de ellos, para lograr a partir de un esquema resultados verídicos en torno a la investigación. 
TABLA 8

Propuesta de estrategias educativas e investigativas para la educación formal en la comunidad

\begin{tabular}{cll}
\hline \multicolumn{2}{l}{ Propuesta 1. Sensibilización de la problemática ambiental de los humedales } \\
\hline $\begin{array}{l}\text { Objetivo: conocer y valorar la problemática ambiental de los humedales y la importancia de su conservación } \\
\text { para el bienestar humano }\end{array}$ \\
\hline \multicolumn{1}{c}{ Población objetivo: estudiantes de secundaria } & \multicolumn{1}{c}{ Estrategias } \\
\cline { 2 - 3 } & $\begin{array}{l}\text { l. Ofrecer charlas y conferencias especializadas sobre la } \\
\text { problemática de los humedales }\end{array}$ & $\begin{array}{l}\text { Cantidad de estudiantes } \\
\text { capacitados }\end{array}$ \\
\cline { 2 - 3 } $\begin{array}{c}\text { Sensibilización } \\
\text { sobre la } \\
\text { problemática }\end{array}$ & $\begin{array}{l}\text { 2. Salidas pedagógicas (desarrollo de talleres en campo } \\
\text { sobre servicios ecosistémicos que permitan a los } \\
\text { estudiantes reconocer las relaciones entre bienestar }\end{array}$ & $\begin{array}{l}\text { Encuestas y entrevistas a } \\
\text { estudiantes que permitan } \\
\text { evaluar el nivel de } \\
\text { humano y conservación de los ecosistemas) }\end{array}$ \\
\cline { 2 - 3 } & $\begin{array}{l}\text { 3. Conmecimiento sobre los } \\
\text { servicios ecosistémicos }\end{array}$ \\
\hline & $\begin{array}{l}\text { Humedales (jornadas ambientales, como caminatas, } \\
\text { siembra de árboles, foros, expresiones artísticas, etc.) }\end{array}$ & $\begin{array}{l}\text { Registro fotográfico de } \\
\text { actividades } \\
\text { conmemorativas }\end{array}$ \\
\hline
\end{tabular}

TABLA 8 (CONT.)

Propuesta de estrategias educativas e investigativas para la educación formal en la comunidad

\begin{tabular}{lll}
\hline Propuesta 2. Incorporación de la temática ambiental de los humedales en los currículos escolares \\
\hline $\begin{array}{l}\text { Objetivo: crear escenarios y oportunidades para el desarrollo de proyectos escolares orientados a la } \\
\text { conservación y uso sostenible de los servicios ecosistémicos de los humedales }\end{array}$ & \\
\hline \multicolumn{1}{l}{ Población objetivo: estudiantes básica y media } & Indicadores \\
\hline & Estrategias & \\
& $\begin{array}{l}\text { 1. Articular los PRAES al desarrollo de proyectos } \\
\text { orientados al uso y la conservación de los servicios } \\
\text { ecosistémicos (evaluación de servicios, soberanía } \\
\text { alimentaria, conocimiento tradicional, etnobotánica, }\end{array}$ & $\begin{array}{l}\text { Cantidad de PRAES } \\
\text { ejecutados }\end{array}$ \\
\cline { 2 - 3 } $\begin{array}{c}\text { 2. Incorporar la } \\
\text { temática de los } \\
\text { humedales a } \\
\text { nivel curricular }\end{array}$ & $\begin{array}{l}\text { 2. Crear un concurso con diferentes categorías que } \\
\text { incentive a los estudiantes a desarrollar y presentar } \\
\text { propuestas artísticas vinculadas a la temática de humedales } \\
\text { (mejor video o reportaje ambiental, mejor fotografía }\end{array}$ & $\begin{array}{l}\text { Concurso creado. } \\
\text { presentadas de propuestas }\end{array}$ \\
& $\begin{array}{l}\text { 3. Crear medios de comunicación (emisoras estudiantiles, } \\
\text { periódico estudiantil) para la divulgación y promoción de } \\
\text { temas, estudios y noticias de carácter ambiental que }\end{array}$ & Medios creados \\
& vinculen la temática de los humedales & \\
\hline
\end{tabular}


TABLA 8 (CONT.)

Propuesta de estrategias educativas e investigativas para la educación formal en la comunidad

\begin{tabular}{|c|c|c|}
\hline \multicolumn{3}{|c|}{ Propuesta 3. Creación de semilleros de investigación } \\
\hline \multicolumn{3}{|c|}{$\begin{array}{l}\text { Objetivo: favorecer el desarrollo de competencias investigativas en los estudiantes y crear semilleros de } \\
\text { investigación en la temática de humedales }\end{array}$} \\
\hline \multicolumn{3}{|c|}{ Población objetivo: estudiantes de $9 .^{\circ}$ a $11 .^{\circ}$ grado. } \\
\hline \multirow{8}{*}{$\begin{array}{l}\text { 3. Semilleros de } \\
\text { investigación }\end{array}$} & Estrategias & Indicadores \\
\hline & $\begin{array}{l}\text { 1. Conformación de grupos escolares de observadores de } \\
\text { aves que apoyen actividades de monitoreo y registro }\end{array}$ & \multirow{7}{*}{$\begin{array}{l}\text { Semilleros conformados. } \\
\text { Cantidad de } \\
\text { investigaciones ejecutadas }\end{array}$} \\
\hline & $\begin{array}{l}\text { 2. Conformación de grupos de investigación en servicios } \\
\text { ecosistémicos y bienestar humano }\end{array}$ & \\
\hline & 3. Conformación de grupos de investigación en & \\
\hline & producción sostenible (desarrollo de iniciativas & \\
\hline & productivas sostenibles para la zona: huertos orgánicos, & \\
\hline & desarrollo de bioabonos, mercados agroecológicos y & \\
\hline & campesinos, fincas demostrativas, etc.) & \\
\hline
\end{tabular}

Fuente: elaborada por Saúl Eduardo Henao y Diego Fernando Carabalí, 2018

\section{Discusión de resultados}

\section{Diagnóstico de la situación ambiental}

El diagnóstico de la situación ambiental de los humedales Cauquita y Guarinó permitió identificar y caracterizar los principales aspectos biofísicos, sociales, económicos, culturales y políticos asociados con ellos. De igual forma, se pudo establecer que las principales fuerzas impulsoras de cambio en los ecosistemas de humedal en el Valle del Cauca han sido la expansión del monocultivo de caña de azúcar, las políticas y desarrollos urbanísticos, el desconocimiento de su problemática y la carencia de mecanismos que permitan establecer acuerdos y consensos entre las partes involucradas para lograr una aplicación efectiva de los planes de manejo ambiental.

Se pudo establecer también que la conservación de los humedales es prioritaria para el bienestar de las comunidades locales, ya que el flujo de servicios ecosistémicos es clave en aspectos como la subsistencia y la producción de alimentos, seguridad material y ambiental y el acceso a soluciones paliativas naturales para afecciones menores, como fiebres, dolores de estómago, indigestiones y resfriados, a través del uso y mantenimiento de más de quince especies de plantas de uso medicinal, como ruda, hierbabuena, menta, anís, romero, pronto alivio, paico, uña de gato, anamú, nacedero, borrachero, jengibre, totumo, papunga y hierba mora.

\section{Evaluación y comparación de la calidad de los servicios ecosistémicos de los humedales Cauquita y Guarinó}

El instrumento utilizado para la identificación, caracterización y evaluación de la calidad de los servicios mostró ser una herramienta consistente que permitió establecer las relaciones entre las acciones y actividades impactantes en los humedales y el suministro de servicios ecosistémicos.

Asimismo, el instrumento facilitó priorizar y calificar once servicios ecosistémicos de los que depende el bienestar de las comunidades locales. Se pudo observar que los impactos que más contribuyen al deterioro 
de las condiciones ecológicas de los humedales son la alteración del flujo hídrico, el uso intensivo del suelo, el agotamiento del recurso hídrico, el vertimiento de efluentes agrícolas y domésticos, la fragmentación y deterioro del hábitat, la eutrofización y la pérdida de especies útiles.

\section{Propuesta educativa}

Se pudo constatar que uno de los aspectos que más influye en la duración y permanencia de las actividades que impactan sobre la provisión de servicios ecosistémicos es el desconocimiento de su problemática y la carencia de mecanismos y espacios que permitan articularla e integrarla en los currículos académicos y como parte de la vida cotidiana de las personas.

Igualmente, se pudo identificar que actualmente en los colegios no existen iniciativas para la conformación de semilleros de investigación o grupos ecológicos desde los cuales se contribuya a visibilizar y generar conciencia sobre la problemática de los humedales, así como también sobre su gran cantidad de contribuciones al bienestar humano a través de la provisión de servicios ecosistémicos.

Lo anterior representa una oportunidad para difundir y posicionar actividades que impliquen una valoración positiva de la naturaleza y refuercen el vínculo humano con ella.

\section{Conclusiones}

El diagnóstico ambiental permitió concluir que los humedales Cauquita y Guarinó son espacios de alta importancia para la conservación de biodiversidad, ya que ofrecen refugio y mantienen condiciones de hábitat para diversas especies, especialmente aves, las cuales representan el grupo faunístico más diverso, con 101 especies registradas, y en las cuales hay un importante potencial turístico, que puede impulsar la conservación de los humedales y al mismo tiempo generar ingresos para las poblaciones locales.

Los humedales son importantes dinamizadores de la cultura y representan espacios de encuentro para las comunidades; en ellos se mantienen y se trasmiten tradiciones y saberes que les permiten mantenerse unidos como comunidad.

Se hace importante complementar las propuestas educativas actuales con temáticas acerca del deterioro y la importancia de la preservación de estas áreas del departamento y de todo el país como reservorios de flora y fauna y como parte del pulmón del mundo.

Sería ideal que las instituciones educativas, especialmente las cercanas a los humedales, sensibilizaran a los estudiantes haciendo visitas dirigidas, con el objetivo de sembrar en los jóvenes la semilla de la investigación.

\section{Referencias}

Alcaldía de Medellín. (2012).Propuesta para la gestión integral de la biodiversidady los servicios ecosistémicos en Medellín. Medellín: Instituto Humbolt.

Arana, V. (2015). Análisis y valoración de los servicios de los ecosistemas de humedales asociados al río León (Urabá antioqueño - Colombia). Su relación con el sistema hídrico subterráneo y con el bienestar humano (tesis de maestría). Universidad Nacional de La Plata, Argentina. Recuperado de http://bit.ly/2HLUfmO

Asociación Colombiana de Ingeniería Sanitaria y Ambiental (Acodal). (1991). Memoria Seminario Internacional de Impacto Ambiental. Metodologías Empresas Públicas de Medellín. Agosto 27 al 30.

Balvanera, P. (2012). Los servicios ecosistémicos que ofrecen los bosques tropicales. Revista Ecosistemas, 21(1-2), 136147.

Bonilla-Castro, E., y Rodríguez-Sehk, P. (2005). Más allá del dilema de los métodos de investigación: la investigación en ciencias sociales. Bogotá: Universidad de los Andes y Norma. 
De Groot, R., Wilson, M., y Boumans, R. (2002). A typology for the classification, description and valuation of ecosystem functions, goods and services. Ecological Economics, 41(3), 393-408.

Díaz, S., Fargione, J., Chapin III, F. S., y Tilman, D. (2006). Biodiversity Loss Threatens Human Well-Being. PLoS Biol, 4(8): e277. https://doi.org/10.1371/journal.pbio.0040277

Expósito-Verdejo, M. (2003). Diagnóstico rural participativo. Una guia práctica. Santo Domingo: Centro Cultural Poveda.

González, A., Ramírez, W., Benítez, A., y Moreno, J. (2014). Estudio de los humedales tipo cauce aislado de río Cauca, Jamundi, Colombia. Cali: Poemia.

Haines-Young, R., y Potschin, M. B. (2017). Common International Classification of Ecosystem Services (CICES) V5.1. Guidance on the Application of the Revised Structure.

Hernández, R., Fernández, C., y Baptista, P. (1991). Metodología de la investigación. México: MacGraw Hill.

IGCP 604 (s.f.). Ficha de Diagnóstico Humedales Vinculados a las Aguas Subterráneas, sección Servicios Humedales. Recuperado el 15 de mayo de 2018. http://www.mdp.edu.ar/hidrogeologia/IGCP604/objectives.php

Millennium Ecosystem Assessment. (2005). Ecosystem and Human Well-Being: Synthesis. Washington, D. C.: Island.

Ministerio del Medio Ambiente. (2013). Biodiversidad y servicios ecosistémicos. Bogotá: Instituto Humbolt.

Popó, G. E., y Ruiz, A. M. (2012). La juga como expresión cultural en las comunidades afrocolombianas del corregimiento de Quinamayó, Jamundí, Valle del Cauca (tesis de grado). Universidad Del Valle, Santiago De Cali, Colombia.

Ramsar. (1971). La importancia de los humedales. Recuperado de http://bit.ly/2EIrg11

Restrepo, C., y Naranjo, L. (1987). Recuento histórico de la disminución de humedales y la desaparición de la avifauna acuática en el Valle del Cauca, Colombia. En H. Álvarez, G. Kattan y C. Murcia (eds.), Memorias III Congreso de Ornitología Neotropical, Cali, Colombia.

Rivera, C., Naranjo, L., y Duque, A. (2007). De María a un mar de caña. Imaginarios de naturaleza en la transformación del paisaje vallecaucano entre 1950 y 1970. Cali: Universidad Autónoma de Occidente.

Rojas, M. P. (2015).Vulnerabilidad de humedales altoandinos ante procesos de cambio: tendencias del análisis. Medellín: Universidad de Medellín.

Sáenz, J. (2009). Configuración de una élite política en Cali 1958-1998. Estudios Regionales y Latinoamericanos, 4. Recuperado de http://bit.ly/2MVM7Vq

Senhadji-Navarro, K. (2017). Ecological Status of some Colombian Wetlandsin the Last 15 Years. Bogotá: Universidad Distrital.

Sistema de Información Ambiental de Colombia (SIAC). (2019, 6 de mayo). Sistema de Información Ambiental de Colombia. Recuperado de http://bit.ly/2Z0mILc

Uribe, H. (2014). De ecosistema a socioecosistema diseñado como territorio del capital agroindustrial y del Estadonación moderno en el valle geográfico del río Cauca, Colombia. Revista Colombiana de Sociología, 37(2), $121-$ 157.

Varguillas, C. (2006). El uso de ATLAS.ti y la creatividad del investigador en el análisis cualitativo de contenido upel. Laurus, 12, 73-78.

Vásquez, E. (2001). Historia de Cali en el siglo XX. Sociedad, economia, cultura y espacio. Santiago de Cali: Artes Gráficas del Valle.

Vilardy, S., y González, J. (2011). Repensando la Ciénaga. Nuevas miradas y estrategias para la sostenibilidad en la Ciénaga Grande de Santa Marta. Santa Marta: Universidad del Magdalena y Universidad Autónoma de Madrid.

\section{Notas}

* Artículo de investigación Este artículo es producto del proyecto de investigación Estudio Comparado de los Servicios Ecosistémicos de los Humedales Guarinó y Cauquita, en el Valle del Cauca, financiado con recursos propios, para optar al título de la Especialidad de Educación Ambiental y Desarrollo Sostenible de la Universidad Santiago de Cali. Fue 
Saúl Eduardo Henao Echeverría, et al. Estudio comparado de los servicios ecosistémicos de los ...

ejecutado por Saúl Eduardo Henao Echeverría, Diego Fernando Carabalí Altamirano, Luis Antonio Gonzales Escobar y Luis Carlos Marmolejo.

\section{Licencia Creative Commons CC BY 4.0}

Cómo citar este artículo: Henao Echeverría, S. E., Carabalí Altamirano, D. F., González Escobar, L. A., y Marmolejo, L. C. (2018). Estudio comparado de los servicios ecosistémicos de los humedales Guarinó y Cauquita, en el Valle del Cauca (Colombia). Ambiente y Desarrollo, 22(43). https://doi.org/10.11144/Jav eriana.ayd22-43.ecse 